\section{Less expansion, more agreement}

Riccardo Giovanelli

\section{We have known for 70 years that the Universe is expanding, but astronomers couldn't agree on how fast. We may be approaching - with $10 \%$ uncertainty - a consensus value. So is one of astronomy's greatest quests finally over?}

E dwin Hubble got it wrong by an order of magnitude ${ }^{1}$, but claimed an uncertainty of $10 \%$. Seventy years and much humble pie later, our knowledge of the value of the current rate of expansion of the Universe, $H_{0}$, may be approaching that degree of precision. At the American Astronomical Society meeting last month ${ }^{\star}$, members of the Hubble Space Telescope Key Project team announced the results of their eight-year search for $H_{0}$.

The determination of $H_{0}$ requires independent measurements of the recession velocities and distances of extragalactic sources. The relative speeds of distant objects are routinely obtained with high precision from Doppler shifts of spectral lines of stellar or interstellar origin; distance measurements are difficult and more uncertain. The first estimates of $H_{0}$ given by Edwin Hubble (Fig. 1) hovered near $500 \mathrm{~km} \mathrm{~s}^{-1} \mathrm{Mpc}^{-1}$. A megaparsec $(\mathrm{Mpc})$ is a unit of distance equivalent to 3.1 million light years; two galaxies separated by $d \mathrm{Mpc}$ recede from each other at $H_{0} d \mathrm{~km} \mathrm{~s}^{-1}$. These ungainly but practical units used by astronomers will be assumed, but explicitly omitted, throughout this article. Had the expansion of the Universe proceeded unchanged at the galloping rate suggested by Hubble, the Big Bang would have taken place less than two billion years ago, making the Earth older than the Universe. In the 1950s, progress in stellar astronomy and the recognition of observational blunders brought estimates of $H_{0}$ down to less than 100, and there it long stayed, tugged between 50 and 100 by raspy conflict for three decades.

The reasons for such large uncertainty stem from the uneasy set of extrapolations required by distance measurements. The operational approach to measuring distances is referred to as the 'cosmological distance ladder', which splices together techniques of progressively greater scope until distances of cosmological interest are reached. With the advent of the Hubble Space Telescope (HST), the opportunity arose to substantially improve the distances to which one of the most important links of the ladder could be applied. Cepheids are

American Astronomical Society Centennial Meeting, Chicago, Illinois, USA, 30 May to 3 June, 1999. bright, regularly variable stars (the period of their variability is correlated with their mean luminosity), which are assumed for the purposes of the distance ladder to be 'standard candles'. Observations of their apparent brightness at different times indicate their distances, and that of their parent galaxies, from Earth. Several hundred Cepheids have been found with the HST, at distances of a few tens of Mpc, mainly by a team led by W. Freedman, R. Kennicutt and J. Mould $\left(\right.$ Key Project group ${ }^{2}$ ). These have provided a much surer foothold on the distance ladder.

For a reliable value of $H_{0}$, the distance ladder needs to be extended farther than the largest distances at which Cepheids can be seen, so that perturbations produced by mass-density fluctuations in the local Universe have a negligible influence on estimates of Hubble expansion. The next rungs of the ladder are provided by a variety of techniques that more often rely on the relationship between rotational speed and luminosity of spiral galaxies, or the surface brightness fluctuations in the images of elliptical galaxies, or the decay in the light emission of supernovae explosions. In the past, these methods generally yielded significantly different values of $H_{0}$, with the lowest estimate coming from supernovae. But the gap is narrowing. Results from three studies by the HST Key Project team give values of $H_{0}=71$ from spiral galaxies (S. Sakai), $H_{0}=69$ from elliptical galaxies (L. Ferrarese) and $H_{0}=68$ from supernovae (B. Gibson). They all reported uncertainties near $10 \%$.

Other groups have used the Cepheid calibration data in different ways. Another supernovae study found $H_{0}=60$ (A. Saha, Carnegie Observatories), whereas $H_{0}=$ 73-79 was obtained from elliptical data ( $\mathrm{J}$. Tonry, Univ. Hawaii), and $H_{0}=77$ was calculated from spirals (B. Tully, Univ. Hawaii and M. Pierce, Indiana Univ.). Another spiral study by a French-Finnish group ${ }^{3}$ has reported $H_{0} \approx 55$. The source of the discrepancies is to be found among uncertainties in the corrections for sampling biases ${ }^{3}$ and in the deviations from smooth Hubble expansion in the local Universe, which can affect inferences of $H_{0}$ from nearby sources by several per cent ${ }^{4,5}$. Furthermore, differences in the chemical compositions of Cepheids, which can produce shifts in the period-luminosity relation ${ }^{6}$, may lead to systematic errors in distance estimates. So, error assessments are still loaded with unknown systematic components. This is well illustrated by the report of a trigonometric distance to the galaxy NGC4258 by radio interferometry (J. Herrnstein, Nat. Radio Astronomy Observatory, New Mexico); it differs by about $15 \%$ from the Cepheid distance to the same galaxy, whereas reported errors of both techniques vary between $4 \%$ and $7 \%$. As systematic effects are better understood, their impact diminishes and the gap between various estimates of $H_{0}$ narrows. At this time, our understanding of these errors seems to hold $H_{0}$ at the limit of, but not below, the threshold of $10 \%$ accuracy.

In the past few years, new sets of distance indicators, which do not rely on cumbersome distance-ladder extrapolations, have delivered increasingly accurate results. The Sunyaev-Zeldovich effect (SZE) depends on a spectral shift in the photons of the cosmic microwave background, produced by their interaction with the hot gas dwelling in the core of galaxy clusters. A joint measurement of the SZE shift and the X-ray emission of intracluster gas yields an estimate of a cluster's distance. Groups using this technique find values of $H_{0}$ ranging from 71 (B. Mason and S. Myers, Univ. Pennsylvania, Philadelphia), to between 57 and 64 (J. Carlstrom, Univ. Chicago). Uncertainties in $H_{0}$ from SZE determinations are about $15 \%$, arising

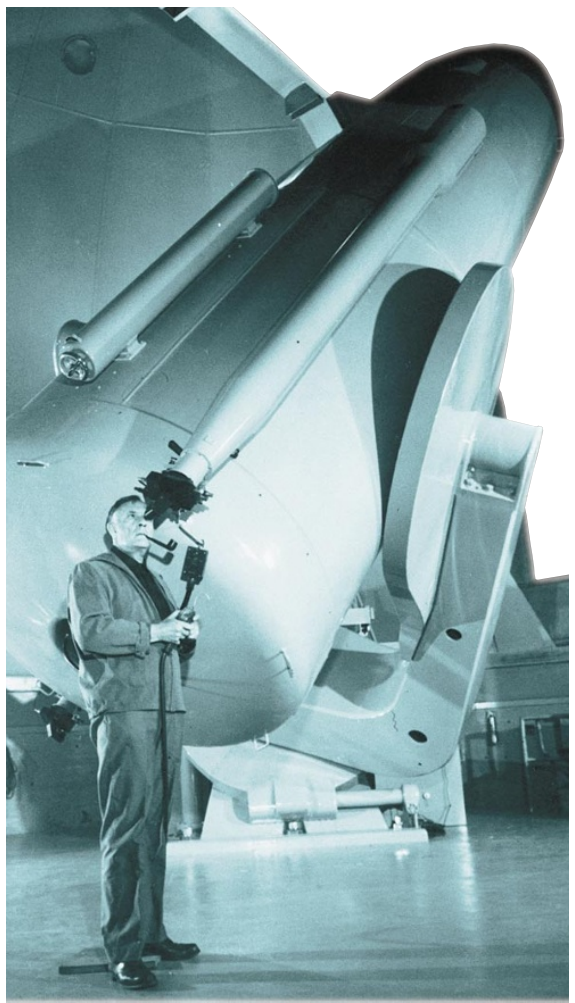

Figure 1 Edwin Hubble, who discovered that the Universe is expanding. Knowing the rate of expansion, the Hubble constant, is essential for determining the age and size of the Universe. 


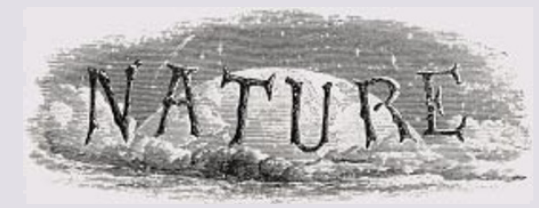

100 YEARS AGO

The Science Section of the Women's Congress was held at the Small Hall in the Westminster Town Hall on Thursday, June 29 ... . The subject of research work was discussed, and stress was laid upon the fact that, inasmuch as the majority of students who take up science do so either as an avenue to a degree or with the idea of earning a livelihood by teaching later on, their training was as a rule insufficient and quite inadequate to permit them to undertake independent original work; whilst on the other hand the demands upon their time made by teaching was so great as to leave practically no leisure for higher work, even when they were qualified to do it. ... It was highly satisfactory to find that, in the open discussion which followed, an attempt on the part of two speakers to introduce the question of vivisection from the anti-vivisectionist point of view was not tolerated by the audience, these speakers being refused a hearing. It is not too much to say that the papers contributed were worthy both of their subjects and their authors, and that there was a refreshing absence of the hackneyed comparison of the relative position and intellectual powers of men and women, which has been such a favourite theme with so many speakers at this Congress.

From Nature 6 July 1899.

\section{YEARS AGO}

Dr. E. Rosen has written a remarkably lively little book, the conclusion of which is that the term 'telescope' was originally devised by John Demisiani of Cephalonia, and made public by Frederick Cesi at the banquet given in honour of Galileo on April 14, 1611. The circumstances may seem to the man of science of to-day to be too minute to warrant the publication of a book; but we must welcome here what the history of science so notably lacks, namely, a painstaking and detailed inquiry into a doubtful point concerning which both contemporaries and historians are at variance. The book scarcely needs to justify itself by its subject-matter, for such is the gusto of its author that the reader is infected by that strange passion that keeps sensible men out of bed after midnight to read detective stories.

From Nature 9 July 1949. principally from the imperfectly known geometry of the clusters. Because SZE can be measured on very distant clusters, the technique samples smooth Hubble expansion (negligibly disturbed by local effects), but its results are dependent on the large-scale geometry of the Universe, which accounts for the spread in the values reported by Carlstrom. An alternative technique that measures time delays between multiple images of a distant source (produced by the gravitational lensing of an intervening mass concentration) is also starting to yield interesting results (P. Schechter, MIT). Findings from a study of lens Q0957 + 561 gives $H_{0}=61$, with an uncertainty of $20 \%$ (A. Romanowsky and C. Kochanek) ${ }^{7}$.

In matter-dominated cosmological models, values of $H_{0}$ above 60 have the embarrassing feature of yielding an age for the Universe since the Big Bang that is exceeded by the oldest stars in our Galaxy, which are approaching an elderly 12 billion years ${ }^{8}$. With the discovery $^{9,10}$ that the expansion of the Universe may be accelerated by dark, perhaps vacuum energy, even relatively high values of $H_{0}$ become consistent with stellar astrophysics, removing this age paradox. Since the 1950s, Allan Sandage in particular has towered over efforts to measure $H_{0}$. At times it seemed like Sandage and Gustav Tammann were holding a tug-of-war at the $60 \mathrm{~km} \mathrm{~s}^{-1} \mathrm{Mpc}^{-1}$ line, against a swelling crowd pulling for the $80 \mathrm{~s}$, 90s and even higher. After several decades of taking this heroic stance, it appears to me that Sandage and Tammann have a fair claim to victory - it may not be 43 (ref. 11), but 66 is starting to look pretty good.

Riccardo Giovanelli is in the Department of

Astronomy, Cornell University, Ithaca,

New York 14853, USA.

e-mail: riccardo@astrosun.tn.cornell.edu

1. van den Bergh, S. in The Extragalactic Distance Scale (eds Livio, M., Donahue, M. \& Panagia, N.) 1 (Cambridge Univ. Press, 1997). http://oposite.stsci.edu/pubinfo/pr/1999/19/key.html

3. Ekholm, T. et al. http://xxx.lanl.gov/abs/astro-ph/9904372

4. Zehavi, I., Riess, A. G., Kirshner, R. P. \& Dekel, A. Astrophys. J. 503, 483-491 (1998).

5. Giovanelli, R., Dale, D., Haynes, M. P., Hardy, E. \& Campusano, L. Astrophys. J. (in the press).

6. Kennicutt, R. et al. Astrophys. J. 498, 181-194 (1998).

Romanowsky, A. \& Kochanek, C. Astrophys. J. 516, 18-36 (1999).

8. Chaboyer, B., Demarque, P., Kernan, P. J. \& Krauss, L. M.

Astrophys. J. 494, 96-110 (1998).

9. Perlmutter, S. et al. Astrophys. J. 517, 565-590 (1999)

10. Riess, A. G. et al. Astron. J. 116, 1009-1038 (1998).

11. Sandage, A. Astrophys. J. 402, 3-14 (1993).

\section{Aerodynamics \\ How flies fly}

\section{Robin Wootton}

nsects are by far the oldest, most numerous and smallest flying machines. Writing in Science, Dickinson and colleagues ${ }^{1}$ now fill a large gap in our understanding of their unconventional - but effective - aerodynamic techniques. Using a flapping, robotic fruitfly (Drosophila), Dickinson et al. show how transient bursts of lift can be generated by the wings as they rotate at the top and bottom of each stroke. Their work not only contributes to this lively field of study, but goes a long way towards providing a general theory of insect flight.

Like helicopters, but unlike conventional aircraft, insects support their weight, propel themselves and control their attitude and manoeuvres by actively moving their wings relative to their body and accelerating a precisely directed mass of air: directly down if hovering; obliquely down if flying forwards, backwards or sideways. Helicopters rotate their wings around a more-or-less vertical shaft. Insects, which have no rotary bearings, oscillate and twist their wings, generally ensuring that as much of the stroke-cycle as possible is creating usefully directed aerodynamic force.

Conventional aerodynamic theory was built on fixed wings in a steady airflow. It has been plain for a quarter of a century that the airflow around insects is anything but steady, and that they make extensive use of aerodynamic tricks to gain remarkably high levels of lift. In the past few years a good deal of research has been directed towards uncovering the mechanisms involved.

A flying insect beats its wings down relative to its body; then twists them backwards around their longitudinal axes; beats them up; and twists them forwards. Downstroke and upstroke - which are actually usually oblique, sometimes even horizontal - are the translational phases of the stroke. The brief periods of twisting at the top and bottom of the stroke are the rotational phases.

Wings generate lift by creating vortices: whirlwind-like circulations of air around a linear core. Crucial among these is the bound vortex, which circulates around the wing itself, speeding up the mass airflow over the upper surface of the wing, slowing it down beneath, accelerating it down behind, and creating a pressure difference between the upper and lower surfaces which tends to suck the wing upwards. The stronger this circulation, the higher the lift; and both increase with the angle of attack - the angle between the wing and the oncoming flow - until the critical stalling angle is reached, when the flow abruptly separates from the upper wing surface and lift disappears.

If a wing is suddenly accelerated at a high angle of attack, stalling can briefly be delayed, and extraordinarily high lift generated, by the formation of another vortex on the upper surface of the wing just behind the 